\title{
Thalamic Reticular Nucleus Activation Reflects Attentional Gating during Classical Conditioning
}

\author{
Kerry McAlonan, Verity J. Brown, and Eric M. Bowman \\ School of Psychology, University of St. Andrews, St. Andrews KY16 9JU, United Kingdom
}

\begin{abstract}
All senses, except olfaction, are routed through the thalamus to cerebral cortex. Thus, the thalamus is often referred to as the sensory gateway to cortex. Located between thalamus and cortex is a thin lamina of neurons called the thalamic reticular nucleus, which may function as an attentional gate. The phenomenon of blocking in classical conditioning provides an opportunity to test whether an attended stimulus activates the thalamic reticular nucleus more than an unattended stimulus: when a second stimulus is presented together with a previously conditioned stimulus, conditioned responding to the second stimulus is inhibited.

Different groups of rats were given conditioning sessions with a single stimulus, a light or a tone, and then given conditioning
\end{abstract}

The thalamic reticular nucleus receives collaterals from both thalamocortical and corticothalamic fibers (Jones, 1975; Ohara and Lieberman, 1985), and therefore it is in a position to gate the flow of information between thalamus and cortex. By virtue of its anatomy and physiological properties, the thalamic reticular nucleus has been implicated in attention (Skinner and Yingling, 1977; Yingling and Skinner, 1977; for review, see Guillery et al., 1998). There is also behavioral evidence to support this view (Friedberg and Ross, 1993; Montero, 1997; Weese et al., 1999). Crick (1984) said that if the thalamus is the gateway to cortex, then the thalamic reticular nucleus is the "guardian of the gateway."

Functional sectors of the thalamic reticular nucleus are defined by the origin of the cortical and thalamic collaterals they receive and by physiology. In the rat, a visual sector is located caudodorsally in the nucleus (Sumitomo et al., 1976; Hale et al., 1982; Coleman and Mitrofanis, 1996; Lozsadi et al., 1996), whereas an auditory sector is ventral to this (Shosaku and Sumitomo, 1983). These subsectors of the reticular nucleus have extensive inhibitory interconnectivity and therefore lateral inhibition could enhance the relay of relevant (attended) information while attenuating the relay of irrelevant (unattended) information. Because the organization of the thalamic reticular nucleus suggests that it may function as an attentional gate, we tested this idea by using a marker of neuronal activation, the presence of Fos protein, during a behavioral task in which attention is directed to one stimulus and not another, Kamin's (1969) blocking procedure. In this procedure, a stimulus that reliably predicts reward evokes a conditioned response. A second stimulus, introduced after conditioning but presented simultaneously with the first, is redundant and therefore results in no conditioned response. This second stimulus is referred to as the "blocked stimulus."

Received May 31, 2000; revised Aug. 22, 2000; accepted Sept. 12, 2000.

This work was supported by the Medical Research Council (UK) project grant number G9531294 (E.M.B.) K.M. received a Biotechnology and Biological Sciences Research Council (UK) Special Studentship. We thank Mary Latimer for advice and assistance with immunohistochemistry, Dr. Patrick Pallier for helpful discussion, and the staff of the School of Psychology Animal House and Workshop.

Correspondence should be addressed to Dr. Verity J. Brown, School of Psychology, University of St. Andrews, St. Andrews KY16 9JU, Scotland, UK. E-mail: vjb@ st-and.ac.uk.

Copyright (C) 2000 Society for Neuroscience $0270-6474 / 00 / 208897-05 \$ 15.00 / 0$ sessions with compound (light and tone) stimuli. Blocking was confirmed using probe trials of single stimulus presentations. After a final test session of compound stimulus presentations, the brains were processed for the presence of Fos protein. Here we show that Fos-positive neurons were more numerous in the sector of the thalamic reticular nucleus associated with the attended conditioned stimulus than in the sector associated with the unattended stimulus. Thus, we provide evidence for an involvement of the thalamic reticular nucleus in selective attention.

Key words: attention; thalamus; thalamic reticular nucleus; classical conditioning; rat; blocking

Using cytochrome oxidase as a metabolic marker, it has been demonstrated that secondary auditory cortex responds to an auditory conditioned stimulus predictive of footshock, but not if the stimulus is blocked by previous conditioning to a visual stimulus (Poremba et al., 1997). Metabolic differences between the two conditions were observed in structures in which the convergence of auditory and somatosensory input is modulated by visual input. Thus, the authors concluded that blocking was not attributable to inattention to the blocked stimulus, supporting instead an associative explanation of blocking (Rescorla and Wagner, 1972). Nevertheless, their data do not preclude the possibility that blocking has an attentional component. Attentional theories of blocking (Mackintosh, 1975; Solomon, 1977; Pearce and Hall, 1980; Holland and Gallagher, 1993) propose that limited attentional resources are directed to the conditioned stimulus. Because the blocked stimulus conveys no additional relevant information, it is unattended. If the thalamic reticular nucleus mediates selective attention, labeling of Fos protein will be restricted to the sector of the thalamic reticular nucleus associated with the attended conditioned stimulus and not in the sector of thalamic reticular nucleus associated with the blocked, unattended, stimulus.

\section{MATERIALS AND METHODS}

Subjects and apparatus. Fifteen experimentally naïve male Lister hooded rats (Charles River, Margate, UK) were pair-housed. The colony was maintained on a $12 \mathrm{hr}$ light/dark cycle (lights on 7:00 A.M.) with water available ad libitum in the home cage and food restricted to $15-20 \mathrm{gm} / \mathrm{d}$, provided after testing. Procedures were licensed under the UK Animals (Scientific Procedures) Act, 1986. Testing was conducted in standard Skinner boxes, with a food hopper between two fixed levers (Campden Instruments, Sileby, UK).

Procedure. An appetitive blocking procedure, with food reward, was used (Table 1). After conditioning to a single stimulus and exposure to the compound stimulus, rats were given probe trials of alternating presentations of single stimuli under extinction, to verify blocking. Thus, there were $12 \mathrm{~d}$ of conditioning sessions, comprising 120 stimulus-reward pairings per day. The conditioned stimulus, presented for $1 \mathrm{sec}$, was a light in the food hopper (light, $n=5$ ) or an $800 \mathrm{~Hz}, 70$ decibel tone (tone, $n=6$ ) from a speaker located in the ceiling of the chamber, or the light and tone together (both, $n=4)$. The reward was a $50 \%$ sucrose pellet $(45 \mathrm{mg}$; BioServ, Frenchtown, NJ), delivered into the hopper by automatic dispenser coincident with the offset of the conditioned stimulus. Interstimulus intervals were randomized between 1 and $30 \mathrm{sec}$. On day 13 of training, there was a single session of 70 presentations of the compound stimulus followed by reward. Blocking was assessed the following day by 30 alternating presen- 
Table 1. Experimental procedure

\begin{tabular}{lllll} 
Group & $\begin{array}{l}\text { CS conditioning } \\
(12 \times 120 \text { trials })\end{array}$ & $\begin{array}{l}\text { Compound conditioning } \\
(70 \text { trials })\end{array}$ & $\begin{array}{l}\text { Probe trials } \\
(30 \text { alternating } \\
\text { CS-no reward })\end{array}$ & $\begin{array}{l}\text { Test } \\
(105 \mathrm{~min})\end{array}$ \\
\hline Light & Light + reward & & $\begin{array}{l}\text { Light, tone, } \\
\text { Light, tone, } \\
\text { Light, tone, }\end{array}$ & \\
Tone & Tone + reward & & $\ldots$ & Light $/$ tone + reward \\
Both & Light $/$ tone + reward & Light $/$ tone + reward & $\ldots$
\end{tabular}

tations of the light and tone alone, with no reward. Responses at the food hopper were recorded, and responses in the $1 \mathrm{sec}$ preceding the stimulus were subtracted from responses during the stimulus, so that positive values would indicate greater responding during the stimulus, and negative values would indicate greater responding before the stimulus. The final test session, which lasted $105 \mathrm{~min}$, consisted of presentations of the compound stimulus followed by reward.

Immunohistochemistry. Immediately after the final session, the brains were processed for the presence of Fos protein. To prevent nonspecific Fos elevation, for the $12 \mathrm{hr}$ preceding the final test, rats were held in darkness with minimal exposure to auditory and visual stimuli. Rats were carried to the darkened testing room in covered cages and immediately placed in the operant chambers. Fos protein synthesis follows mRNA expression 30-45 min after stimulation and has a half-life of $2 \mathrm{hr}$. Therefore, the rats were anesthetized immediately after the final test session and transcardially perfused with saline followed by $4 \%$ paraformaldehyde.

One in four $50 \mu \mathrm{m}$ sections were cut and put into PBS. Sections were treated as follows: 30 min wash in $20 \%$ sucrose solution (in PBS), two rinses in PBS followed by a 60 min wash in blocking solution $(100 \mathrm{ml}$ of PBS, $20 \mathrm{ml}$ of goat serum, and $1 \mathrm{ml}$ of Triton X-100), two PBS rinses, 48 hr incubation in primary c-fos antibody (Ab-5; 1:20,000; Oncogene Research Products, Cambridge, MA), five PBS rinses, 45 min incubation period on a shaker in Vector Laboratories (Peterborough, UK) IgG solution $(5 \mu \mathrm{l} / \mathrm{ml}$; Vectastain rabbit ABC kit), five PBS rinses, $45 \mathrm{~min}$ incubation in $\mathrm{ABC}$ complex (antibody diluting solution, substrate $\mathrm{A}$ and substrate B, each $20 \mu \mathrm{l} / \mathrm{ml}$ ) on a shaker, and five PBS rinses, incubated for $2-10 \mathrm{~min}$ in diaminobenzidine peroxidase substrate (Sigma Fast 3-3' diaminobenzidine tetrahydrochloride with metal enhancer tablet sets; Sigma-Aldrich, Poole, UK).

The regions of interest were the visual sector and the auditory sector of the thalamic reticular nucleus. Based on electrophysiology (Shosaku and Sumitomo, 1983) and anatomy (Pinault and Deschenes, 1998), these re-

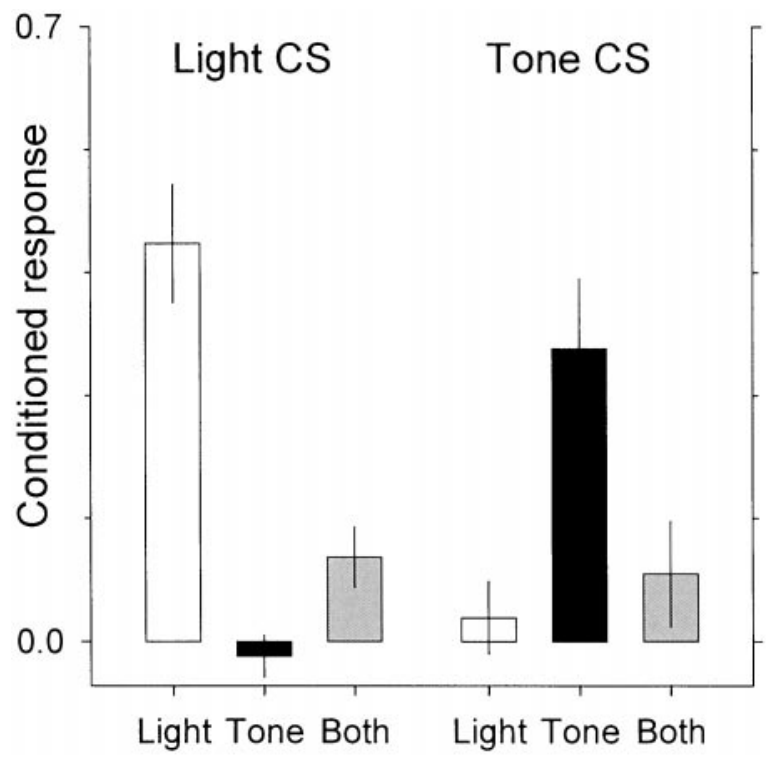

Figure 1. Conditioned responding was assessed in probe trials in which the stimuli-light or tones-were presented alone and not followed by food reward. Responses in the $1 \mathrm{sec}$ before stimulus onset were subtracted from responses during the $1 \mathrm{sec}$ stimulus. The left panel shows responding to the light; the right panel shows responding to the tone. The rats were preconditioned, either with the light (white bars), the tone (black bars), or the compound (gray bars) stimulus. The number of hopper entries was highest when the preconditioned stimulus was presented and lowest when the blocked stimulus was presented. Intermediate values were observed for rats trained with the compound stimulus. gions are located approximately between bregma -3.1 and $-4.1 \mathrm{~mm}$, with the visual sector located dorsal to the auditory sector. Therefore, we counted all neurons stained positively for Fos protein in the dorsal 50\% and ventral $50 \%$ of the thalamic reticular nucleus on 15 sections between -2.7 and $-4.1 \mathrm{~mm}$, but used counts from the sections between approximately bregma -3.1 and $4.1 \mathrm{~mm}$ to obtain means for visual (dorsal $50 \%$ ) and auditory (ventral 50\%) sectors.

\section{RESULTS}

The probe trials confirmed that the procedure resulted in a conditioned response to the stimulus presented during the conditioning sessions and not to the second stimulus. Rats conditioned to the compound stimulus showed intermediate levels of conditioned responding to either stimulus alone (Fig. 1; interaction of group and stimulus, $\left.F_{(2,12)}=24.7, p<0.01\right)$.

Modality-specific subsectors of the thalamic reticular nucleus were activated according to the attended stimuli. Neurons stained positively for Fos protein were more numerous in the sector of the thalamic reticular nucleus associated with the conditioned, attended, stimulus (Figs. 2, 3). Thus, rats conditioned to light showed activation of visual thalamic reticular nucleus, whereas rats conditioned to tone showed activation of the auditory thalamic reticular nucleus. Rats who had received previous conditioning to the compound stimulus showed activation of both visual and auditory

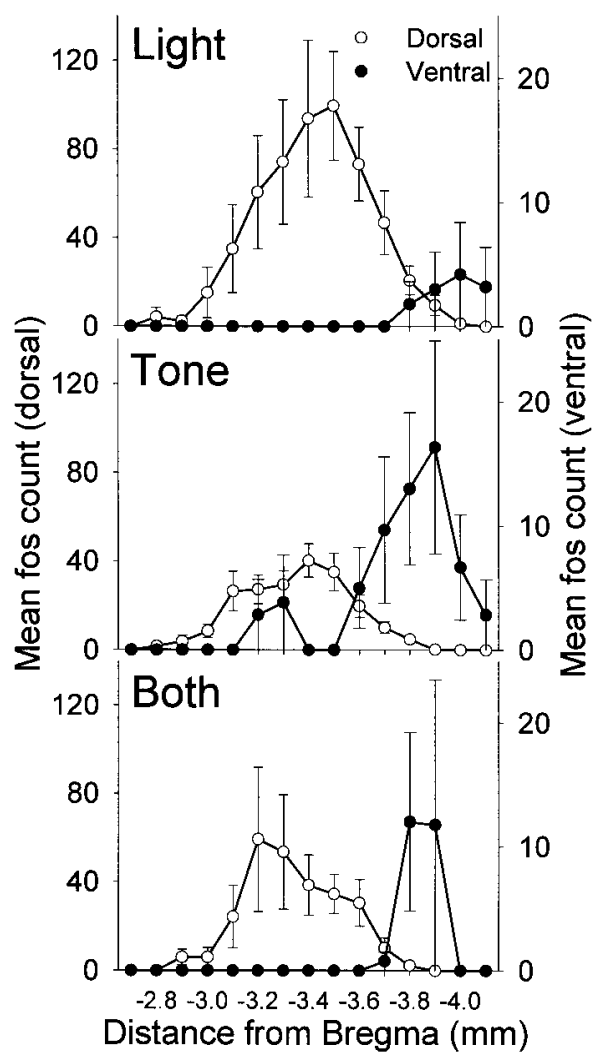

Figure 2. Mean counts of Fos-positive neurons in the dorsal $50 \%$ and ventral $50 \%$ of the thalamic reticular nucleus on sections between bregma -2.7 and $-4.1 \mathrm{~mm}$ for each group. 


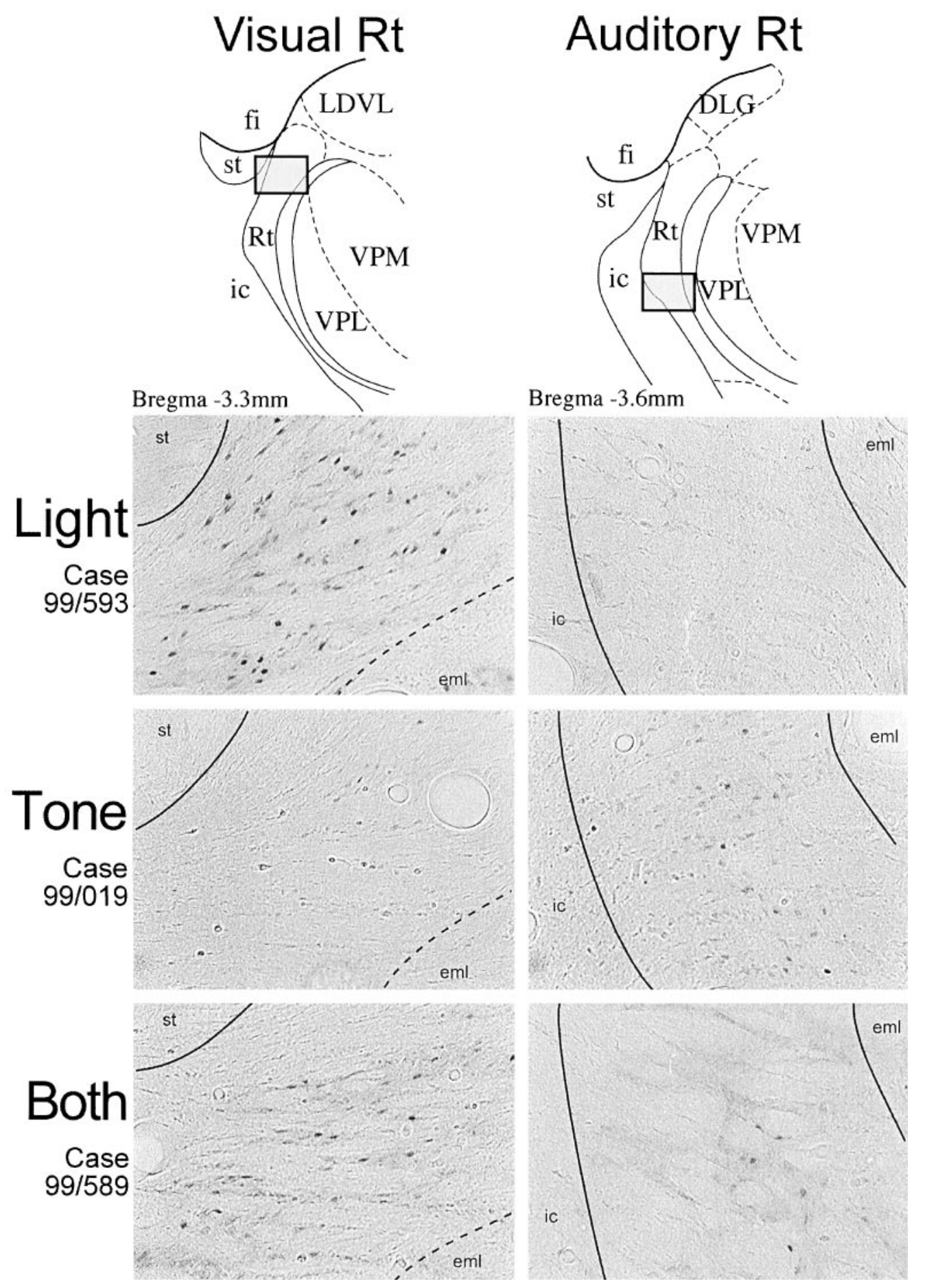

Figure 3. Coronal sections through the thalamic reticular nucleus of the same hemisphere of one rat from each group, showing Fos-positive neurons in different sectors of the thalamic reticular nucleus according to the group. Fos staining was seen in the dorsal (visual) portion of the thalamic reticular nucleus in the light-conditioned, but not in the tone-conditioned, group. The converse pattern of activity was found in the ventral and more posterior (auditory) portion of the thalamic reticular nucleus. In the compound stimulusconditioned rats, Fos-positive neurons were found in both regions. On the test day, all rats received the same, compound, stimulus presentations. Abbreviations from Paxinos and Watson (1997): Rt, thalamic reticular nucleus; $f i$, fimbria; $s t$, stria terminalis; $i c$, internal capsule; eml, external medullary lamina; $L D V L$, laterodorsal nucleus ventrolateral division; $V P M$, ventral posteromedial thalamic nucleus; $V P L$, ventral posterolateral thalamic nucleus. thalamic reticular nucleus (interaction of group and thalamic reticular sector, $\left.F_{(2,12)}=4.0, p<0.05\right)$.

Across rats in all groups, there was a positive correlation between magnitude of conditioned responding to the light and the number of Fos-labeled neurons in the visual thalamic reticular nucleus (Fig. 4; partial correlation, removing variability caused by auditory conditioned responding, $r=0.45$, df $=12, p=0.053$, one-tailed). There was a stronger positive correlation between the magnitude of auditory conditioned responses and the number of Fos-positive neurons in the auditory thalamic reticular nucleus (partial correlation, removing variability caused by visual conditioned responding, $r=0.62$, $\mathrm{df}=12, p<0.01$, one-tailed). Thus, in addition to the mean differences between groups, the number of Fos-positive neurons in each sensory sector predicts the magnitude of behavioral effects in individual animals.

\section{DISCUSSION}

Activation of the thalamic reticular nucleus was observed in rats attending to conditioned stimuli in a blocking procedure. The activation was specific to the sector of the thalamic reticular nucleus associated with the sensory modality of the conditioned stimulus. There was less activation of the sector of the thalamic reticular nucleus associated with the sensory modality of a blocked stimulus, which did not elicit a conditioned response. The degree of Fos labeling in the thalamic reticular nucleus correlated with the magnitude of the conditioned response to the conditioned stimulus. This result supports the view that the thalamic reticular nucleus is involved in the processing of sensory information (Hartings et al., 2000) but demonstrates that this processing is modulated by attention. 


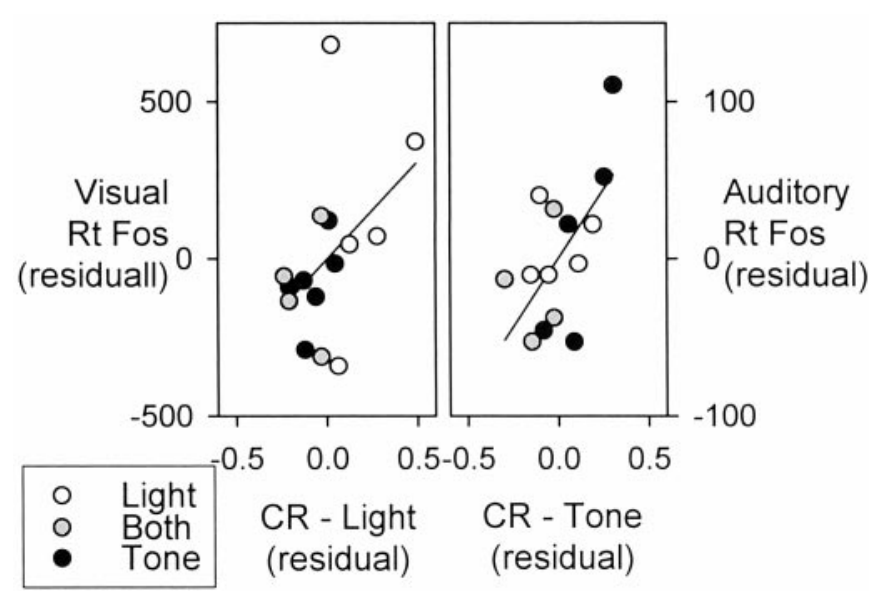

Figure 4. Plots showing the residual conditioned response against the residual Fos count. The visual responses (visual conditioned response and the mean Fos count in the visual portion of the thalamic reticular nucleus) were predicted from the auditory responses and vice versa. The observed responses were then subtracted from predicted responses, to obtain the residual. Thus, variability in the residuals of the visual (or auditory) responses is independent of variability in the auditory (or visual) responses.

The three groups of rats-light, tone, and both light and tonediffered only according to the stimuli to which they had received before conditioning. On the test day, all rats were treated the same and received the same repeated presentations of the compound light-tone stimulus. The possibility that the rats, in attending and responding to the conditioned stimulus, might fail to orient to the second stimulus was considered. To avoid this objection, the light stimulus was presented within the food hopper, to which all the rats oriented and approached when the compound stimulus was presented. The tone was presented from an omnidirectional speaker, located above the rat in the chamber. Thus, the stimuli impinging on the sensorium of the rats would not differ as a function of the rats orienting behavior, so that neural differences can reasonably be expected to reflect differences in attention and not sensation. It should also be noted that no attempt was made to restrict other stimuli within the chamber during the test session: a ventilation fan was on throughout and neither the dispenser nor the hopper panel was silent.

Montero (1997) demonstrated selective c-fos activation in the visual sector of the thalamic reticular nucleus in normal rats exploring a complex novel environment and in the somatosensory sector of the thalamic reticular nucleus in functionally blind rats, dependent on tactile cues to explore. Montero (1997) suggested that sectors of the thalamic reticular nucleus actively compete for limited attentional resources. It is also plausible to suggest that the sectors of the thalamic reticular nucleus might compete to provide limited attentional resources and thus the thalamic reticular nucleus subserves selective attention. Montero (2000) has recently shown that Fos labeling in the thalamic reticular nucleus, but not the geniculate nucleus, is dependent on primary visual cortical input.

Rhythmic burst firing in the thalamus, including the reticular nucleus, is prevalent during periods of neural synchronization, such as during slow wave sleep, deep anesthesia, or absence seizures (Steriade et al., 1986, 1993a,b) and is thought to represent a temporary detachment of relay cells from their sensory inputs. In periods of cortical desynchrony, the thalamic reticular nucleus fires in a tonic mode. Guido and Weyand (1995) demonstrated that arrhythmic burst firing in thalamic relay cells in awake animals may support better signal detection by elevating the signal-to-noise ratio and suggested that the functional implications of burst versus tonic firing in the awake animal may relate to orienting versus focal attention. This suggestion is given further support by recent work of Ramcharan et al. (2000), who suggest that burst firing in the awake animal provides an attentional "wake-up call." The switch from burst to tonic firing in thalamic relay cells may be modulated by descending cortical projections and by the cholinergic innervation of the thalamus and thalamic reticular nucleus (McCormick and Prince, 1986; Marks and Roffwarg, 1991; Kim and McCormick, 1998). The thalamic reticular nucleus inhibits thalamic relay cells (French et al., 1985; Cox et al., 1997; Kim et al., 1997), which would be consistent with the suggestion that sensory input activates the thalamic reticular nucleus, which in turn inhibits and changes the firing mode of the thalamic relay cells. Therefore, it is parsimonious to suggest the Fos-labeling seen here reflects neuronal excitation in the sector of the thalamic reticular nucleus associated with the attended modality.

The specificity of the labeling of thalamic reticular nucleus supports the view that this nucleus is more than a component of the sensory relay but rather acts as an attentional gate or filter. These data do not demonstrate that the mechanism of blocking is attentional, however they do provide support for the suggestion that blocking has an attentional component, changing the processing of stimuli according to the associative value of the stimulus.

\section{REFERENCES}

Coleman KA, Mitrofanis J (1996) Organization of the visual reticular thalamic nucleus of the rat. Eur J Neurosci 8:388-404.

Cox CL, Huguenard JR, Prince DA (1997) Nucleus reticularis neurons mediate diverse inhibitory effects in thalamus. Proc Natl Acad Sci USA 94:8854-8859.

Crick F (1984) Function of the thalamic reticular complex: the searchlight hypothesis. Proc Natl Acad Sci USA 81:4586-4590.

Friedberg EB, Ross DT (1993) Degeneration of rat thalamic reticular neurons following intrathalamic demoic acid injection. Neurosci Lett 151:115-119.

French CR, Sefton AJ, Mackay-Sim A (1985) The inhibitory role of the visually responsive region of the thalamic reticular nucleus in the rat. Exp Brain Res 57:471-479.

Guido W, Weyand T (1995) Burst responses in thalamic relay cells of the awake behaving cat. J Neurophysiol 74:1782-1786.

Guillery RW, Feig SL, Lozsadi DA (1998) Paying attention to the thalamic reticular nucleus. Trends Neurosci 21:28-32.

Hale PT, Sefton AJ, Bauer LA, Cottee LJ (1982) Interrelations of the rats thalamic reticular and dorsal lateral geniculate nuclei. Exp Brain Res 45:217-229.

Hartings JA, Temereanca S, Simons DJ (2000) High responsiveness and direction sensitivity of neurons in the rat thalamic reticular nucleus to vibrissa deflections. J Neurophysiol 83:2791-2801.

Holland PC, Gallagher M (1993) Amygdala circuitry in attentional and representational processes. Trends Cogn Sci 3:65-73.

Jones EG (1975) Some aspects of the organization of the thalamic reticular complex. J Comp Neurol 162:285-308.

Kamin LJ (1969) Predictability, surprise, attention and conditioning. In: Punishment and aversive behaviour (Campbell BA, Church RM, eds), pp 279-296. New York: Appleton-Century-Crofts.

Kim U, McCormick DA (1998) The functional influence of burst and tonic firing mode on synaptic interactions in the thalamus. J Neurosci 18:9500-9516.

Kim U, Sanchez-Vives MV, McCormick DA (1997) Functional dynamics of GABAergic inhibition in the thalamus. Science 278:130-134.

Lozsadi DA, Gonzalez-Soriano J, Guillery RW (1996) The course and termination of corticothalamic fibres arising in the visual cortex of the rat. Eur J Neurosci 8:2416-2427.

McCormick DA, Prince DA (1986) Acetylcholine induces burst firing in thalamic reticular neurones by activating a potassium conductance. Nature 319:402-405.

Mackintosh NJ (1975) A theory of attention: variations in the associability of stimuli with reinforcement. Psychol Rev 82:276-298.

Marks GA, Roffwarg HP (1991) Cholinergic modulation of responses to glutamate in the thalamic reticular nucleus of the anaesthetised rat. Brain Res 557:48-56.

Montero VM (1997) C-fos induction in sensory pathways of rats exploring a novel environment: shifts of active thalamic reticular sectors by predominant sensory cues. Neuroscience 76:1069-1081.

Montero VM (2000) Attentional activation of the visual thalamic reticular nucleus depends on "top-down" inputs from the primary visual cortex via corticogeniculate pathways. Brain Res 864:95-104.

Ohara PT, Lieberman AR (1985) The thalamic reticular nucleus of the adult rat: experimental anatomical studies. J Neurocytol 14:365-411.

Paxinos G, Watson C (1997) The rat brain in stereotaxic coordinates, Ed 3. New York: Academic.

Pearce JM, Hall G (1980) A model for Pavlovian learning: variations in the effectiveness of conditioned but not of unconditioned stimuli. Psychol Rev 106:532-552. 
Pinault D, Deschenes M (1998) Projection and innervation patterns of individual thalamic reticular axons in the thalamus of the adult rat: a three-dimensional graphic and morphometric analysis. J Comp Neurol 391:180-203.

Poremba A, Jones D, Gonzalez-Lima F (1997) Metabolic effects of blocking tone conditioning on the rat auditory system. Neurobiol Learn Mem 68:154-171.

Ramcharan EJ, Gnadt JW, Sherman SM (2000) Burst and tonic firing in thalamic cells of unanesthetized behaving monkeys. Vis Neurosci 17:55-62.

Rescorla RA, Wagner AR (1972) A theory of Pavlovian conditioning: Variations in the effectiveness of reinforcement and nonreinforcement In: Classical conditioning II: current research and theory (Black $\mathrm{AH}$, Prokasy WF, eds) pp 64-99. New York: Appleton-Century-Crofts

Shosaku A, Sumitomo I (1983) Auditory neurons in the rat thalamic reticular nucleus. Exp Brain Res 49:432-442.

Skinner JE, Yingling CD (1977) Reconsideration of the cerebral mechanisms underlying selective attention and slow potential shifts. In: Attention, voluntary contraction and event-related cerebral potentials Progress in clinical neurophysiology, Vol 1 (Desmedt JE, ed), pp 30-69. Basel: Karger.

Solomon PR (1977) Role of the hippocampus in blocking and conditioned inhibition of the rabbit's nictating membrane response. J Comp Physiol Psychol 91:407-417.

Steriade M, Domich L, Oakson G (1986) Reticularis thalami neurons revisited: activity changes during shifts in states of vigilance. J Neurosci 6:68-81.

Steriade M, Contreras D, Curro DR, Nunez A (1993a) The slow $(<1 \mathrm{~Hz})$ oscillation in reticular thalamic and thalamocortical neurons: scenario of sleep rhythm generation in interacting thalamic and neocortical networks. J Neurosci 13:3284-3299.

Steriade M, McCormick DA, Sejnowski TJ (1993b) Thalamocortical oscillations in the sleeping and aroused brain. Science 262:679-685.

Sumitomo I, Nakamura M, Iwama K (1976) Location and function of the so-called interneurons of the rat lateral geniculate body. Exp Neurol 51:110-123.

Weese GD, Phillips JM, Brown VJ (1999) Attentional Orienting is impaired by unilateral lesions of the thalamic reticular nucleus in the rat. J Neurosci 19:1035-10139.

Yingling CD, Skinner JE (1977) Gating of thalamic input to cerebral cortex by nucleus reticularis thalami. In: Attention, voluntary contraction and event-related cerebral potentials. Progress in clinical neurophysiology, Vol 1 (Desmedt JE, ed), pp 70-96. Basel: Karger. 